\title{
Contaminação Ambiental de Áreas de Lazer e Recreação por Parasitos de Importância Médica
}

\section{Environmental Contamination of Leisure and Recreation Areas by Parasites of Medical Importance}

\author{
Vinícius Corrêa ${ }^{1}$ \\ Eduardo Faria Porto Arantes ${ }^{2}$ \\ Cecília Gomes Barbosa ${ }^{3}$ \\ César Gómez-Hernández ${ }^{4}$ \\ Karine Rezende-Oliveira ${ }^{5}$
}

\section{RESUMO}

Objetivo: Avaliar a presença de parasitos em amostras de fezes e de solo colhidas em diferentes áreas no Município de Ituiutaba-MG. Metodologia: Foram visitadas 17 praças e coletadas um total de 85 amostras de fezes, analisadas pelas técnicas parasitológicas de flutuação, formol-éter e sedimentação espontânea. As amostras de solo foram colhidas em 20 locais entre praças e clubes, sendo cinco amostras em cada local. As amostras foram acondicionadas em sacos formando um "pool do local", e identificadas com data e temperatura do solo e encaminhadas para análise parasitológica. Resultados: Do total de amostras de fezes coletadas $23,5 \%$ (20/85) estavam positivas, sendo $68 \%(15 / 20)$ apresentaram formas evolutivas de Ancylostoma spp e $9 \%$ das amostras (2/20) continham ovos de Dipylidium caninum e/ou de Toxocara spp. Quanto à presença de protozoários, $9 \%(2 / 20)$ das amostras tinham cistos e/ou trofozoíto de Giardia duodenalis e 4,5\% (1/20) apresentaram oocistos de coccídeos. Do total de amostras de solo coletadas, 70\% (14/20) dos locais positivaram para formas parasitárias de helmintos, sendo que $78,5 \%(11 / 14)$ apresentaram formas evolutivas de Ancylostoma spp , 64,3\% (9/14) de Toxocara spp., $14,3 \%$ (2/14) Strongyloides stercolaris, 7,2\% (1/14) D. caninum ou Hymenolepis nana ou Spirometra mansonoides. Conclusão: $\mathrm{O}$ encontro de formas parasitárias nestes locais destaca a necessidade de ações de promoção em saúde, colocando em prática o conceito de saúde única.

\section{DESCRITORES}

Contaminação Ambiental. Saúde Pública. Parasitoses. Prevalência.

\begin{abstract}
Objective: To evaluate the presence of parasites in stool and soil samples collected in different areas in the municipality of Ituiutaba-MG. Methodology: 17 town squares were visited and a total of 85 stool samples were collected, analyzed by parasitological flotation techniques, formaldehyde-ether, and spontaneous sedimentation. Soil samples were collected at 20 locations between town squares and clubs, with five samples at each location. The samples were conditioned in bags forming a "pool of the place", and identified with date and soil temperature, sent for parasitological analysis. Results: Of the total stool samples collected, $23.5 \%(20 / 85)$ were positive, with $68 \%$ (15/20) presenting evolutionary forms of Ancylostoma spp and $9 \%$ of the samples $(2 / 20)$ presenting eggs. Dipylidium caninum and/or Toxocara spp. regarding the presence of protozoa, $9 \%$ $(2 / 20)$ of the samples contained cysts and/or trophozoites from Giardia duodenalis and $4.55 \%$ (1/20) presented coccidia oocysts. Of the total soil samples collected, $70 \%(14 / 20)$ of the sites were positive for parasitic forms of helminths, with $78.5 \%$ (11/14) showing evolutionary forms of $A$. caninum, $64.3 \%$ (9/14) of Toxocara spp., 14.3\% (2/14) Strongyloides stercolaris, $7.2 \%(1 / 14) D$. caninum or Hymenolepis nana or Spirometra mansonoides. Conclusion: The encounter of parasitic forms in these places highlights the need for health promotion actions, putting the concept of Unique Health into practice.
\end{abstract}

\section{DESCRIPTORS}

Environmental Contamination. Public Health. Parasites. Prevalence.

${ }^{1}$ Graduado em Ciências Biológicas. Laboratório de Ciências Biomédicas, Universidade Federal de Uberlândia, Campus Pontal, Ituiutaba, Minas Gerais, Brasil.

${ }^{2}$ Graduado em Ciências Biológicas. Laboratório de Ciências Biomédicas, Universidade Federal de Uberlândia, Campus Pontal, Ituiutaba, Minas Gerais, Brasil.

${ }^{3}$ Doutora em Medicina Tropical e Infectologia. Pós-Doutoranda do Programa de Pós-Graduação em Medicina Tropical e Infectologia, com Área de Concentração em Parasitologia e Imunologia Aplicadas. Laboratório de Imunologia, Universidade Federal do Triângulo Mineiro, Uberaba, Minas Gerais, Brasil.

${ }^{4}$ Doutor em Medicina Tropical e Infectologia. Pós-Doutorando do Programa de Pós-Graduação em Medicina Tropical e Infectologia, com Área de Concentração em Parasitologia e Imunologia Aplicadas. Laboratório de Imunologia, Universidade Federal do Triângulo Mineiro, Uberaba, Minas Gerais, Brasil.

${ }^{5}$ Doutora em Medicina Tropical e Infectologia, com Área de Concentração em Parasitologia e Imunologia Aplicadas. Docente da Universidade Federal de Uberlândia, Campus Pontal, Ituiutaba, Minas Gerais, Brasil. 
$\mathrm{O}_{\mathrm{r}}^{\mathrm{s}}$ parques públicos e as áreas de recreação são ambientes comumente frequentados por adultos e crianças, geralmente acompanhados por seus animais de estimação. Estudos em vários países apontam uma alta taxa de contaminação de solo nestes ambientes, pois animais infectados eliminam os parasitos através das fezes, aumentando o risco de transmissão zoonótica e a (re) infecção por outros animais ${ }^{1}$.

O solo nesses ambientes comportase como um reservatório, onde se localizam parasitos em estágios não infectantes, que juntamente com os fatores abióticos do local, como o calor, a umidade e as altas taxas de oxigênio, favorece a diferenciação de alguns parasitos, atingindo assim os estágios infectantes, gerando questões de cunho epidemiológico ${ }^{2,3}$.

As alterações climáticas influenciam diretamente no ciclo de vida de alguns parasitos, aumentando ou diminuindo a sobrevivência dos estágios evolutivos no ambiente. Fatores como o aumento da umidade favorece a sobrevivência de ovos, larvas e cistos/oocistos do parasito. A temperatura elevada acelera o seu desenvolvimento no ambiente e nos hospedeiros ectodérmicos, no entanto, diminui a sobrevivência de ovos, larvas, cistos e oocistos ${ }^{4}$.

Os cães são considerados hospedeiros para alguns parasitos entre eles a Giardia duodenalis, Toxocara canis, Cryptosporidium spp, Ancylostoma spp Echinococcus granulosus, Dipylidium caninum e Toxoplasma gondii $^{3,5}$. As fezes dos cães abrigam vários estágios infecciosos destes parasitos sendo uma importante fonte de infecção para animais e humanos ${ }^{3,5}$.
A transmissão aos seres humanos ocorre por meio da forma fecal-oral (contato pessoa a pessoa ou animal a pessoa), pela ingestão de ovos, cistos ou oocistos evidenciando casos ligados ao hábito de higiene dos indivíduos, pois podem se alimentar de hortaliças mal lavadas, não higienizar as mãos, após frequentarem locais possivelmente contaminados com formas parasitarias, dado que, crianças e jovens possuem práticas geofágicas (comer terra) e onicofágicas (roer unha), possibilitando a ingestão direta de parasitos ${ }^{6}$. Além disso, destaca-se a infecção por penetração ativa de larvas na pele do hospedeiro de alguns helmintos que estejam no solo 2,7.

As infecções por protozoários intestinais e helmintos são frequentes e constituem um problema de saúde pública, principalmente em países tropicais e subtropicais em desenvolvimento. Mesmo com o avanço em medidas de saneamento e higiene, estima-se que cerca de três bilhões de pessoas estejam infectadas com parasitos intestinais em todo mundo 8,9

Áreas de recreação, como as praças e os parques, constituem um local de lazer para os habitantes da cidade. A contaminação parasitária do solo por fezes de animais nestes locais é considerada um indicador direto de risco de contágio, aos quais os moradores são expostos ${ }^{2}$. Considerando a importância epidemiológica destes parasitos encontrados em cães e a possibilidade de poluição do solo por formas evolutivas infectantes, o objetivo deste estudo foi identificar os parasitos em locais públicos do Município de Ituiutaba, Minas Gerais, para propor medidas preventivas a fim de promover a conscientização dos usuários, donos de animais e órgãos responsáveis. 


\section{METODOLOGIA}

Área de estudo

O Município de Ituiutaba localiza-se na microrregião do Triangulo Mineiro/Alto Paranaíba, no interior de Minas Gerais, Brasil, apresentando uma população em torno de 104.671 habitantes. O município conta com área total de $2.598,046 \mathrm{~km}^{2}$, estabelecida na latitude $18^{\circ} 58^{\prime} 8^{\prime} \mathrm{S}$ e longitude $49^{\circ} 27^{\prime} 54^{\prime}$ W caracterizado por biomas do Cerrado e Mata Atlântica além de clima quente úmido, tropical no período de inverno, com época de chuva bem definidas entre outubro à abril e estação de seca de maio à setembro. A temperatura média é $14^{\circ} \mathrm{C}$ à $30^{\circ} \mathrm{C}$, precipitação pluviométrica anual média de $1.470 \mathrm{~mm}$ e uma umidade anual de $72 \%$.

Coleta das amostras de fezes

Foram realizadas coletas semanais de amostras de fezes de cães em 17 praças públicas do Município de Ituiutaba, Minas Gerais no período de agosto a setembro de 2018 a agosto a setembro 2019. Em cada praça, foram escolhidos até sete pontos principais de coleta, considerando as amostras que estavam mais próximas dos assentos públicos, em locais com areia, terra e gramado e calçadas onde as pessoas circulam, totalizando 85 amostras. Durante a coleta foi descartado a parte superior da amostra e aquela que estivesse em contato com o solo, com o objetivo de evitar a contaminação. Em dois momentos foi possível colher amostra recém-emitida, com características diarreicas, por se tratar de um animal que estava presente no momento da coleta.
O material foi coletado, identificado, verificando se amostra era de cão (não foi colhida amostra em areia ou coleção de terra, evitando assim amostras de fezes de gatos) e transportado até o Laboratório de Ciências Biomédicas (LACBIM) da Universidade Federal de Uberlândia-Campus Pontal para serem processados. Parte das amostras de fezes (5 gramas) foi colocada em recipiente limpo e estéril e processada para a realização da técnica de flutuação com solução saturada de sacarose (solução de Sheater) ${ }^{10}$. Foi realizado o método de sedimentação por centrifugação em formol-éter e sedimentação espontânea para a procura de ovos de helmintos. A análise ocorreu em microscopia óptica com os aumentos de 100x e 400x.

Coleta de amostras de solo

As coletas de amostras de solos foram realizadas em diferentes locais de lazer e recreação: praças públicas, clubes poliesportivos e escolas que possuíam parques e areia, localizados em todo o perímetro urbano da cidade.

Com o auxílio de um tubo de PVC de 5 centímetros $(\mathrm{cm})$ de comprimento $\times 6 \mathrm{~cm}$ de diâmetro e uma pá de jardinagem foram colhidas amostras de solo. Foi introduzido o tubo a uma profundidade de $5 \mathrm{~cm}$, coletando cinco amostras de solo em pontos diferentes, formando um quadrante e uma amostra central. Em seguida, as amostras foram colocadas em saco plástico identificado com o número, local e data, formando uma amostra única. As coletas foram padronizadas no período da manhã e avaliadas pelo programa AcuuWeather (State College, Pensilvânia, 
EUA). Foi aferida a temperatura do solo, a cada coleta, determinado por um termômetro digital infravermelho.

O material foi encaminhado ao Laboratório de Ciências Biomédicas do Instituto de Ciências Exatas e Naturais do Pontal, da Universidade Federal de Uberlândia, Campus Pontal, para processamento e análise.

No laboratório, as amostras foram misturadas e retirados 200 gramas (g), para a análise, realizada logo após a coleta.

Foram realizadas as técnicas de sedimentação por centrifugação em formol-éter, Ritchie, sedimentação espontânea e a técnica Rugai ${ }^{11}$ baseada em termohidrotropismo e a análise ocorreu em microscopia óptica com os aumentos de 100x e 400x.

Foram consideradas para análise ao menos duas lâminas de cada amostra realizada por dois observadores independentes e a identificação das formas parasitárias foi realizada com base das informações de atlas, artigos científicos e livros didáticos.

Análise estatística

Para a análise dos dados obtidos nos exames de fezes e de solo foi utilizado o $\chi 2$ de Pearson, com nível de significância $p<0,05$. Nas análises, foi utilizado o programa Stastistica 8.0 (Statsoft, Inc).

\section{RESULTADOS}

Do total de 85 amostras coletadas $23,5 \%$ (20/85) estavam positivas, contendo formas evolutivas de helmintos e protozoários.

Considerando a distribuição das amostras de fezes coletadas em relação às praças contaminadas nos setores do município, $80 \%$ (4) estavam no setor Norte; $75 \%$ (3) estavam no setor Sul; no setor Oeste $100 \%$ (3) e no setor Leste $60 \%$ (3).

Em relação às amostras positivas, $68,2 \%(15 / 20)$ apresentaram ovos de Ancylostoma spp, além disso, em $9 \%$ (2/20) foram observadas cápsulas ovígeras de Dipylidium caninum, em 9\% (2/20) ovos Toxocara spp. Quanto a presença de protozoários, $9 \%(2 / 20)$ das amostras continham cistos e trofozoíto de Giardia duodenalis e $4,5 \%(1 / 20)$ apresentaram oocistos de Coccideos (Figura 1).

O Ancylostoma spp foi o parasito mais frequente em amostras de fezes colhidas em todas as regiões, principalmente nos setores Norte e Oeste $(p<0,05)$. O Dipylidium caninum foi encontrado apenas no setor Leste. O Toxocara spp. foi encontrado nos setores Norte e Leste. A Giardia duodenalis foi encontrada no setor Norte e Sul e oocistos de coccídeos apenas no setor Sul. No setor Leste foi encontrada a maior diversidade de parasitos (Ancylostoma spp, Dipylidium caninum e Toxocara spp.) quando comparados aos outros setores (Figura 1).

Foram coletadas amostras de solo em 20 áreas (cinco amostras/local) de lazer e recreação no Município de Ituiutaba, Minas Gerais totalizando 100 amostras por local. A temperatura ambiente média do local foi de $26,6^{\circ} \mathrm{C}$ e umidade em média de $38,1 \%$. A temperatura média do solo foi de $29,9^{\circ} \mathrm{C}$ (Figura 2).

Do total de amostras de solo coletadas em praças e áreas de recreação, 70\% (14/20) 
Figura 1. Percentagem (\%) de parasitos encontrados em fezes de cães coletadas de praças públicas da Cidade de Ituiutaba, Mias Gerais

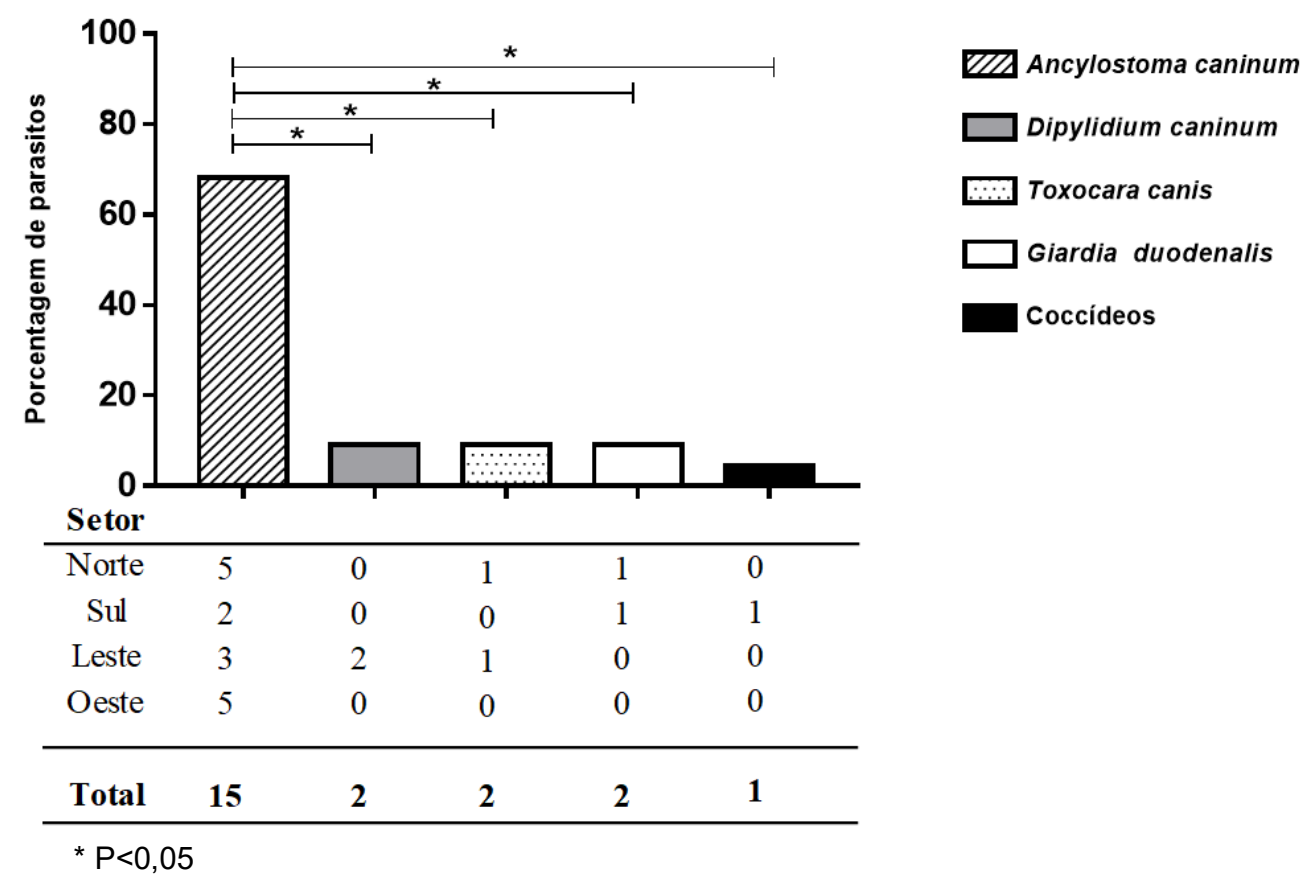

Figura 2. Média da representação climática da Temperatura $\left({ }^{\circ} \mathrm{C}\right)$ e Umidade $(\%)$ por setores municipais durante a coleta de fezes e amostras de solo no Município de Ituiutaba, Minas Gerais

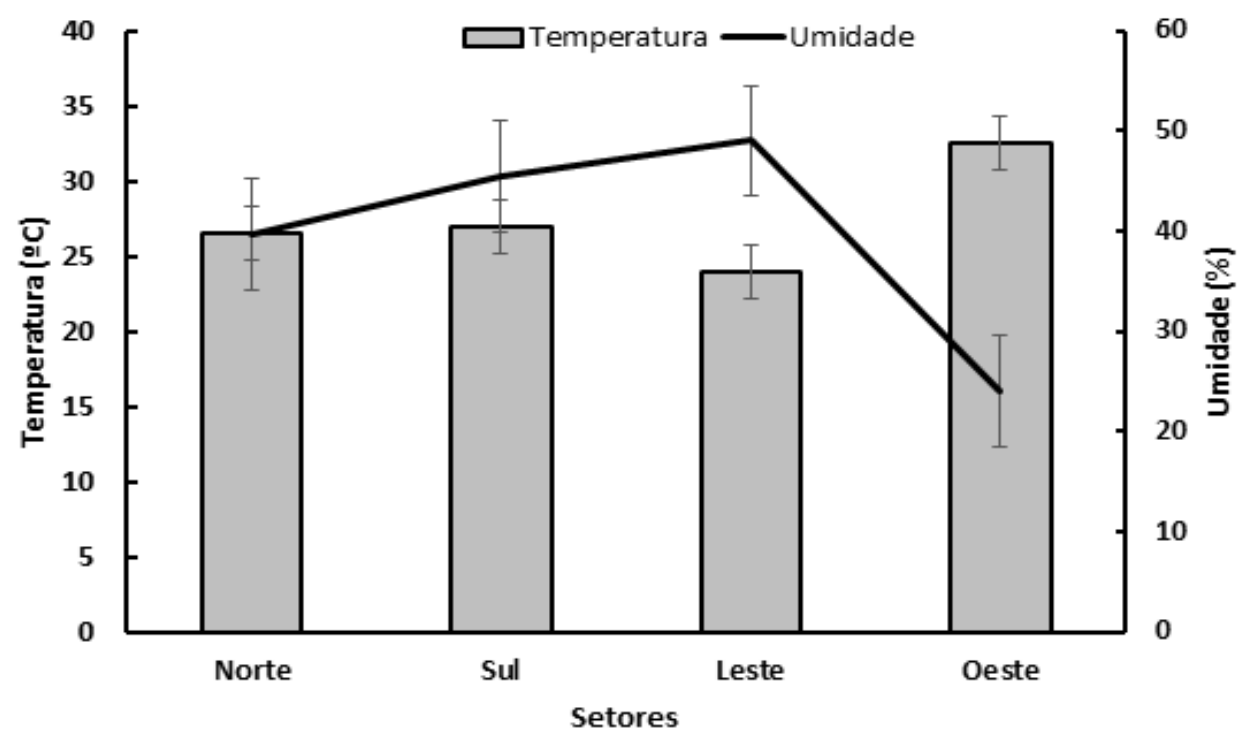


dos locais apresentaram positividade para formas parasitárias de helmintos.

Observando as amostras de solo oriundos dos locais que foram positivos para formas parasitárias de helmintos, verificou-se que 78,57\% (11/14) apresentaram larva filarioide, rabditoide e ovos, sendo algumas amostras com ambas as formas (larvas/ovos) de Ancylostoma spp. As larvas foram identificadas observando o esôfago característico da forma evolutiva (Figura 3). A presença de ovos de Toxocara spp. foi descrita em $64,3 \%$ (9/14) dos locais com amostras positivas (Figura 3 ). Além disto, notou-se a ocorrência das formas evolutivas (larva filarioide, identificada pelo primórdio genital) de Strongyloides stercolaris em 14,3\% (2/14) dos locais (Figura 3 ).

Foi observada a presença de ovos de Dipylidium caninum (7,2\%), Hymenolepis nana $(7,2 \%)$ e Spirometra mansonoides (7,2\%). Detectou-se o oocisto de coccídeo (Toxoplasma gondii) em duas amostras (Figura 3).

Foi observado contaminação do solo com múltiplas formas parasitárias em 71,4\% (10/14) das amostras positivas, notando-se a presença de Ancylostoma spp em 90\% (12/14), Toxocara spp. em 80\% (11/14), Spirometra mansonoides em 10\% (2) e Strongyloides stercolaris em 20\% (3/14) das amostras positivas.

Figura 3. Percentagem (\%) de parasitos encontrados em amostras de solo coletadas de praças públicas e áreas de recreação da Cidade de Ituiutaba, Minas Gerais

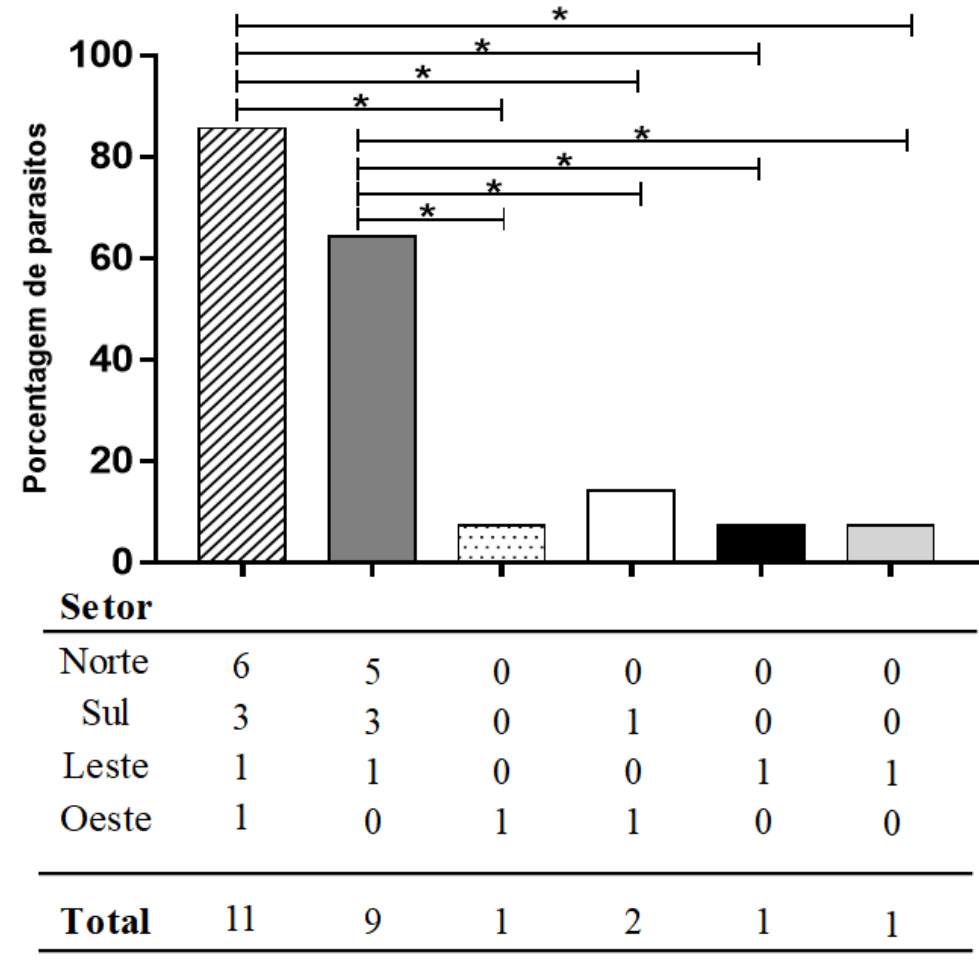

${ }^{*} \mathrm{P}<0,05$

\section{ZII Ancylostoma spp \\ Toxocara canis \\ Dipylidium caninum \\ Strongyloides stercoralis \\ Hymenolepis nana \\ Spirometra mansonoides}




\section{DISCUSSÃO}

A Cidade de Ituiutaba, Minas Gerais onde o estudo foi realizado é caracterizada por ser uma zona climática benéfica para o desenvolvimento dos helmintos e protozoários. As praças e áreas de recreação são ambientes comumente frequentadas pela população, geralmente acompanhados de seus animais de estimação e por animais errantes. A ocorrência de algumas formas evolutivas destes parasitos nestes locais pode gerar um grande problema de saúde pública.

A contaminação do solo por formas infectantes de parasitos intestinais é um fator de risco de infecção mais importante para humanos e animais ${ }^{12}$. Foi observada, nas amostras de solos de todas as regiões do município, a presença de múltiplas formas parasitárias $(71,4 \%)$, com uma variedade de espécies de parasitos de importância médico-veterinário coabitando os mesmos locais. Nas amostras de solo da Região Leste, representada por bairros que estão em desenvolvimento, principalmente de infraestrutura, foi observada uma maior variedade de helmintos. Sabe-se que o encontro de formas parasitárias de helmintos no solo representa um risco à saúde humana. As condições ambientais como a umidade elevada e a temperatura entre 25 e $30^{\circ} \mathrm{C}$ favorecem o desenvolvimento e a manutenção dos seus estágios evolutivos. Além disso, a possibilidade de ausência de saneamento básico e de práticas de higiene, contribuem com a dispersão ambiental desses parasitos $^{13,14}$. É importante mencionar que o encontro de parasitos no solo deve considerar a condição imunológica dos indivíduos, além da situação ambiental que podem favorecer o estabelecimento de parasitoses acometendo uma parcela da população carente em saneamento básico e recursos humanos ${ }^{15}$.

Foi possível evidenciar no solo das áreas de recreação uma alta quantidade $(85,7 \%)$ de ovos e larvas (rabditoide e filarioide) de ancilostomídeos. Esta ocorrência foi observada em estudos anteriores com amostras de solo oriundos de locais de recreação como parques infantis, praças públicas e banco de areia ${ }^{1,16-18}$. Melo et al. ${ }^{9}$ verificou a prevalência de $93,2 \%$ deste parasito em amostras de solo próximo a uma escola primária de ensino no Município de Pelotas, Rio Grande do Sul.

Ao serem analisadas as amostras de fezes de cães coletadas nas praças públicas, verificou-se que os achados foram semelhantes aos observados nas amostras de solo em relação a presença de Ancylostoma spp, em que $68,18 \%$ de amostras continham ovos e/ou larvas. As larvas filarioides de Ancylostoma presentes em fezes e no solo infectam o hospedeiro por meio da penetração ativa na pele ${ }^{20}$. A presença deste parasito nas praças tem uma grande importância epidemiológica e de saúde pública, pois, nas amostras analisadas no presente estudo foram identificadas larvas filarioide, ou seja, com potencial de infecção aos animais e aos seres humanos que frequentam o local, desenvolvendo doenças como o Ancilostomíase e a Larva Migrans Cutânea, respectivamente ${ }^{21}$.

No presente estudo foram detectados ovos de Toxocara spp. em 64,3\% (9/14) das praças e áreas de recreação, semelhante ao observado por Cassenote et al. ${ }^{22}$, que 
descreveram $79,3 \%$ de positividade para ovos de Toxocara sp, sendo $25,6 \%$ em praças públicas e 9,2\% em áreas de recreação, no Município de Fernandópolis, São Paulo. Outros achados relatam o encontro de ovos deste parasito em área comunitárias de lazer, como por exemplo, em amostras de solo de creches em Várzea Paulista, São Paulo, com $100 \%$ de prevalência, $66,7 \%$ nas amostras de solo de escolas de ensino fundamental no norte de uma cidade do Espírito Santo, Brasil e $44,5 \%$ em praças no município de São Paulo ${ }^{22-25}$. Em Uberlândia, MG foi observado em um estudo prévio que $23,07 \%$ das amostras de solo coletadas em praças públicas estavam contaminadas por ovos de Toxocara sp. ${ }^{26}$. Muitas vezes o encontro de Toxocara sp em locais públicos está relacionado com a presença de animais errantes, como cães e gatos, favorecendo a persistência do parasito no solo, o que torna um fator de risco para infecção humana e desenvolvimento de Larva Migrans Visceral.

Ao analisar as amostras de fezes de cães recolhidas nestes locais públicos, observou-se uma frequência de 9,09\% para ovos de Toxocara spp. Abaixa prevalência nas amostras coletadas é justificada uma vez que este parasito infecta comumente cães mais jovens do que adultos ${ }^{27,28}$.

A ocorrência de formas evolutivas de Strongyloides stercolaris (14,3\%) no solo, foi semelhante ao registrado no estudo de Ginar et al. ${ }^{29}$, que encontraram uma prevalência de $16,66 \%$ nos solos de praças e parques públicos do Município de Uruguaiana, Rio Grande do Sul, Brasil. Preussler et al. ${ }^{30}$ observaram amostras de fezes caninas recolhidas nas áreas de lazer e a análise mostrou uma taxa de $2,2 \%$ de Strongyloides sp. em Pelotina, Paraná, Brasil, indicando assim, um fator de contaminação do solo, pelas fezes ali oriundas dos animais errantes presentes ao local. A presença de larvas filarioides no solo é um fator de risco para seres humanos, favorecendo a penetração na pele e provável infecção pelo parasito.

Neste trabalho foi detectada uma baixa frequência de ovos (cápsula ovígera) de Dipylidium caninum $(7,14 \%)$ em amostras de solo, conforme descrito por De Oliveira Figueiredo et al. ${ }^{31}$, que encontraramu $3,1 \%$ em amostras das caixas de areia das instituições escolares infantis do Município de Uruguaiana, Rio Grande do Sul, Brasil. O encontro deste parasito em humanos é pouco frequente, mas pode ocorrer principalmente em crianças menores de 12 meses e que estejam em contato com cães e gatos. Desta forma, a presença de ovos no solo sugere a probabilidade de um hospedeiro intermediário, como pulgas, ingerirem estes ovos e se tornarem um reservatório do parasito, sendo adquiridos por outros animais ou até mesmo crianças $^{32}$.

Em relação ás amostras de fezes, nossas observações mostraram que Dipylidium caninum esteve presente em $9 \%$ das amostras, porcentagem elevada quando comparado com os achados em praças públicas nas Cidades de Natal, RN e Itapema, SC onde foi possível encontrar a tênia canina em 1,7\% e 1,9\% nestes locais, respectivamente ${ }^{33,34}$. Ressalta-se o achado a presença deste parasito geralmente é subestimada quando se realiza os exames coproparasitológicos, considerando que seu diagnóstico é baseado no encontro de 
proglotes em fezes frescas ou e que raramente se encontra cápsulas ovígeras nas fezes ${ }^{35}$.

Quanto a presença de ovos de Spirometra mansonoides $(7,2 \%)$ nas amostras de solo coletadas em praças e áreas de recreação sugere-se que esteja relacionado à presença de gatos como fonte de disseminação ${ }^{36}$. É importante mencionar que na área onde foi realizada a coleta das amostras possui coleções de água, como piscinas públicas frequentadas pela população local, sendo detectada a presença de gatos próximo às áreas de coleta, além de ser um local de fácil acesso por animais errantes, o que pode justificar a presença do parasita.

Foi possível observar oocisto de coccídeo (4,5\%) e cistos/trofozoíto de Giardia em 9 9\% das amostras de fezes de cães. As duas amostras diarreicas foram colhidas assim que o animal defecou e pelo método de sedimentação espontânea foi possível visualizar os trofozoítos após 24 horas de suspensão. A Giardia sp é o protozoário que geralmente possui maior prevalência em estudos que analisam fezes caninas. Este resultado está de acordo com Capuano e Rocha ${ }^{37}$ que observaram uma prevalência de $10 \%$ de amostras positivas nas praças do município de Ribeirão Preto, SP e com estudos realizados por Ribeiro et al. ${ }^{38}$ que descreveram que $15,4 \%$ das amostras colhidas nas praças de Belo Horizonte eram positivas para cistos de Giardia spp.

$\mathrm{Em}$ todos os locais onde foram colhidas amostra de solo e de fezes de animais, observou-se a presença significativa de formas parasitarias. A livre circulação de animais, como cães e gatos nos locais públicos, favorece o aparecimento destes parasitos e a sua permanência no meio, em virtude da vulnerabilidade dos animais. Esse fator torna os animais que frequentam esses locais um reservatório das espécies de parasitos com caráter zoonótico ${ }^{18}$

Os resultados deste estudo mostraram a presença de parasitos de caráter zoonótico nas fezes de cães/gatos e no solo das praças públicas e áreas de recreação no Município de Ituiutaba, Minas Gerais. Estes achados evidenciaram o risco de transmissão nesses locais, tornando este um problema de saúde pública.

\section{CONCLUSÃO}

Em virtude dos achados desse estudo considera-se importante o monitoramento do ambiente, administração de vermífugos em cães errantes, juntamente com a criação de campanhas orientando a população para tratamento de cães domésticos. Além disso, análises de amostras de solos de locais para recreação e lazer, para verificar possível contaminação ambiental, são imprescindíveis para que o município tenha conhecimento da presença de parasitos causadores de zoonoses, em locais públicos e de fácil acesso da população, incentivando o desenvolvimento de ações de promoção em saúde nestas regiões. 


\section{REFERÊNCIAS}

1. Simonato G, Cassini R, Morelli S, Di Cesare A, La Torre F, Marcer $F$, et al. Contamination of Italian parks with canine helminth eggs and health risk perception of the public. Prev. Vet. Med. 2019; 172:104788.

2. Khan JS, Provencher JF, Forbes MR, Mallory ML, Lebarbenchon C, McCoy KD. Parasites of seabirds: A survey of effects and ecological implications. Adv. Mar. Biol. 2019; 82:1-50

3. Khan W, Khan J, Khan N, Iqbal R, Ullah A, Ghaffar R, et al. Soil-transmitted helminth infections in school children of three districts of Malakand region, Khyber Pakhtunkhwa, Pakistan. Pak J Pharm Sci. 2019; 32(2):799-803.

4. Pozio E. How globalization and climate change could affect foodborne parasites. Exp. Parasitol. 2020; 208:107807.

5. Khan W, Nisa NN, Ullah S, Ahmad S, Mehmood SA, Khan M, et al. Gastrointestinal helminths in dog feces surrounding suburban areas of Lower Dir district, Pakistan: A public health threat. Braz. J. Biol. 2019; 80(3):511-517.

6. Gawor J, Borecka A, Zarnowska H, Marczynska M, Dobosz $S$. Environmental and personal risk factors for toxocariasis in children with diagnosed disease in urban and rural areas of central Poland. Vet. Parasitol. 2008; 17;155(3-4):217222.

7. Oishi CY, Klisiowicz DDR, Segui R, Koster PC, Carmena $D$, Toledo R, et al. Reduced prevalence of soil-transmitted helminths and high frequency of protozoan infections in the surrounding urban area of Curitiba, Parana, Brazil. Parasite Epidemiol Control. 2019; e00115.

8. Abbaszadeh Afshar MJ, Barkhori Mehni M, Rezaeian M, Mohebali M, Baigi V, Amiri S, et al. Prevalence and associated risk factors of human intestinal parasitic infections: a population-based study in the southeast of Kerman province, southeastern Iran. BMC infectious diseases. 2020; 6;20(12):1-8.

9. Pacheco-Ortega GA, Chan-Perez JI, Ortega-Pacheco A, Guzman-Marin E, Edwards M, Brown MA, et al. Screening of Zoonotic Parasites in Playground Sandboxes of Public Parks from Subtropical Mexico. J. Parasitol. Res. 2019; 2019:7409076. 1-6.

10. Levine ND. Textbook of veterinary Parasitology. Ed. Minneapolis: Burges, 1978; 236p.

11. Rugai E, Mattos T, Brisola AP. Nova técnica para isolar larvas de nematóides das fezes-modificação do método de Baermann. Rev Inst Adolfo Lutz, 1954; 14(1):5-8.

12. Nkouayep VR, Ngatou Tchakounte B, Wabo Pone J. Profile of Geohelminth Eggs, Cysts, and Oocysts of Protozoans Contaminating the Soils of Ten Primary Schools in Dschang, West Cameroon. J. Parasitol. Res. 2017; 7(1):73-80.

13. Heller L. Relationship between health and environmental sanitation in view of the development. Cien. Saude Colet. 1998; 3(2):73-84,

14. Cutolo SA, Matté MH, Rocha AA. Monitoring of parasitological contamination in treated wastewater from activated sludge system. Manag. Environ. Qual. 2006;17(1):43-56.

15. Elliot SJ, Taylor SM, Walter S. Modeling psychosocial effects of exposure to solid waste facilities. Soc. Sci. Med. 1993; 37(6):790-804.

16. Miranda PHS, Bezerra WFL, Castro TMBQ, Gonçalves LdS. Soil contamination of recreation areas of child day care in public Ancylostoma sp. and toxocara sp. in Teresina-PI. Rev Interd. 2015; 8(4):93-98.

17. Corrêa GLB, Moreira WS. Soil contamination due to eggs of Ancylostoma spp. In public parks of the city of Santa Maria, RS, Brazil. Revista da FZVA. 1996; 3(1):18-23.

18. Blaszkowska J, Goralska K, Wojcik A, Kurnatowski P, Szwabe K. Presence of Toxocara spp. eggs in children's recreation areas with varying degrees of access for animals. Ann Agric. Environ. Med. 2015; 22(1):23-27. 
19. Mello CCS, Nizoli LQ, Ferraz A, Chagas BC, Azario WJD, Villela MM. Helminth eggs with zoonotic potential in the vicinity of public schools in southern Brazil. Rev. Bras. Parasitol. 2020; 29(1):e016419.

20. Traversa D, Frangipane di Regalbono A, Di Cesare A, La Torre F, Drake J, Pietrobelli M. Environmental contamination by canine geohelminths. Parasites \& vectors. 2014; 13; 7:67.

21. Ugbomoiko US, Ariza L, Heukelbach J. Parasites of importance for human health in Nigerian dogs: high prevalence and limited knowledge of pet owners. BMC veterinary research. 2008; 4:49.

22. Cassenote AJ, Pinto Neto JM, Lima-Catelani AR, Ferreira AW. Soil contamination by eggs of soiltransmitted helminths with zoonotic potential in the town of Fernandopolis, State of Sao Paulo, Brazil, between 2007 and 2008. Rev. Soc. Bras. Med. Trop. 2011; 44(3):371374.

23. Chen J, Zhou DH, Nisbet AJ, Xu MJ, Huang SY, Li MW, et al. Advances in molecular identification, taxonomy, genetic variation and diagnosis of Toxocara spp. Infect. Genet. Evol. 2012; 12(7):1344-1348.

24. Siqueira LDP, Fontes DAF, Aguilera CSB, Timoteo TRR, Angelos MA, Silva L, et al. Schistosomiasis: Drugs used and treatment strategies. Acta tropica. 2017; 176:179-187.

25. Regis SC, Mendonca LR, Silva Ndos S, Dattoli VC, Alcantara-Neves NM, Barrouin-Melo SM. Seroprevalence and risk factors for canine toxocariasis by detection of specific $\lg G$ as a marker of infection in dogs from Salvador, Brazil. Acta tropica. 2011; 120(1-2):46-51.

26. Costa-Cruz JM, Nunes RS, Buso AG. Presence of Toxocara spp eggs in public squares of Uberlandia city, Minas Gerais, Brazil. Ver. Inst. Med. Trop. São Paulo. 1994; 36(1):39-42.

27. Trillo-Altamirano MP, CARRASCO AJ, CABRERA R. Prevalence of zoonotic enteroparasite helminths and associated Factors in canis familiaris in an urban area of ica city, Peru. Parasitol. latinoam. 2003; 58:136-141.
28. Santos FAG, Yamamura MH, Vidotto O, Camargo PL. Occurrence of gastrointestinal parasites in dogs (Canis familiaris) with acute diarrhea from metropolitan region of Londrina, Parana State, Brazil. Semina: Ciências Agrárias. 2007; 28(2):257-268.

29. Ginar RMB, Galarça RCG, Picavêa JPP, Petry H. Soil contamination levels event due to dogs nematode eggs in squares of Uruguaiana city - RS, Brazil. Revista da FZVA. 2006; 13(1):103-111.

30. Preussler JV, Valentim-Zabott M, Pinto SB, Montanucci CAR. Pesquisa de ovos e larvas de helmintos em áreas de lazer públicas do município de Palotina, PR, Brasil. Ver. Ciênc. Vet. Saúde Pública. 2016; 3:138-141.

31. Figueiredo MiO, Wendt EW, Santos HT, Moreira CM. Survey of parasite eggs in sandboxes of municipal kindergarten schools in Uruguaiana, Brazil. J. Trop. Path 2012; 41(1):36-46.

32. Neira OP, Jofre ML, Munoz SN. Dipylidium caninum infection in a 2 year old infant: case report and literature review. Rev. Chilena Infectol. 2008; 25(6):465-471.

33. Júnior ALFA, Araújo KBS, Medeiros VS. Ocorrência de Parasitas com Potencial Zoonótico em Fezes de Cães Coletadas em Vias Públicas da Cidade De Natal. Revista Humano Ser - UNIFACEX. 2015; 1:52-59.

34. Blazius RD, Emerick S, Prophiro JS, Romao PR, Silva OS. Occurrence of protozoa and helminthes in faecal samples of stray dogs from Itapema City, Santa Catarina. Rev. Soc. Bras. Med. Trop. 2005; 38(1):73-74.

35. Gennari SM, Kasai N, Pena HFJ, Cortez A. Occurrence of protozoa and helminths in faecal samples of dogs and cats from São Paulo city. Braz. J. Vet. Res. Anim. Sci. 1999; 36(2):87-91.

36. Dall'Agnol LP, Otto MA, Silva AS, G. MS. Gastrointestinal parasites in naturally infected cats in the municipality of Santa Maria in Rio Grande do Sul, Brazil. Acta Vet Bras 2010; 4(3):181-184. 
37. Capuano DM, Rocha GM. Parasites with zoonotic potential in dog feces collected from public areas of the city of Ribeirão Preto, SP, Brazil. Rev Bras de Epidem. 2006; 9(1):81-86.

38. Ribeiro LM, Dracz RM, Mozzer LR, S. LW. Soil contamination in public squares in Belo Horizonte, Minas Gerais, by canine parasites in different developmental stages. Rev Inst Med trop S Paulo. 2013; 55(4):229-231.

\section{CORRESPONDÊNCIA}

Karine Rezende de Oliveira

Universidade Federal de Uberlândia-Campus do Pontal Laboratório de Ciências Biomédicas-LACBIM

Rua 20, 1600, Bloco 1A, sala 408

Bairro Tupã, Ituiutaba, Minas Gerais, Brasil CEP:38304-402

E-mail: karinerezende@ufu.br 